\title{
Analysis of Collaborative Driving Effect of Artificial Intelligence on Knowledge Innovation Management
}

\author{
Qi Liu (iD) ${ }^{1,2}$ \\ ${ }^{1}$ City College of Dongguan University of Technology, Dongguan, China \\ ${ }^{2}$ Universiti Utara Malaysia, Sintok, Malaysia \\ Correspondence should be addressed to Qi Liu; liuq@ccdgut.edu.cn
}

Received 5 November 2021; Revised 20 December 2021; Accepted 3 January 2022; Published 19 January 2022

Academic Editor: Rahman Ali

Copyright (C) 2022 Qi Liu. This is an open access article distributed under the Creative Commons Attribution License, which permits unrestricted use, distribution, and reproduction in any medium, provided the original work is properly cited.

Artificial intelligence (AI) plays a connotative driving role in cross-border knowledge and factor identification of innovation management. This study explores the collaborative driving effects and influence factors of artificial intelligence on knowledge innovation management. Based on the artificial intelligence application data in knowledge innovation management from 2011 to 2020, this paper adopts the synergy model and the adiabatic elimination to empirically test the influence mechanism and key factors of AI for knowledge innovation management. The results showed that artificial intelligence had a significant impact on dynamic elements, capacity elements, environmental elements of knowledge flow, and stock management. AI made endogenous impacts on the promotion of knowledge flow ability and network diffusion. AI technology promoted the formation of the original technological advantages of innovation and had obvious automatic recognition function for new knowledge, which stimulated the main internal transmission power of knowledge innovation. Furthermore, AI actuated energy level of original innovation and progressive achievements of cutting-edge technology, which mainly came from the deep runoff knowledge of innovation system. Knowledge network affected the transmission efficiency and retention proportion of deep runoff knowledge. The penetration proportion of artificial intelligence determined cooperation space of intelligent industry and application direction of integrated knowledge.

\section{Introduction}

As the technology is racing ahead, artificial intelligence is the main event of science and technology development. Facing with the fact of bursting data, knowledge innovation management has got extensive concerned. It is not only an important guarantee for the development of knowledge economy, but also an essential requirement of high-quality economic construction in the new era. So here comes the questions: what are the collaborative driving effects and influence factors of artificial intelligence on knowledge innovation management? How does artificial intelligence promote knowledge innovation management? Artificial intelligence technology has connotative driving effects on the decomposition and application of cross-border elements of knowledge innovation management and has the function of direction indicator for new technological invention, new industrial direction, and new scientific discovery. Therefore, it is particularly important to investigate the collaborative driving factors and effects of artificial intelligence on knowledge innovation management.

Botega and Da Silva [1] proposed that knowledge innovation management could affect the operation law of all kinds of scientific and technological innovation, and its influencing factors were extremely diverse and complex, which mainly played a leading role in the process of knowledge network and flow [1]. Vikingur et al. [2] adopted a regression model to show that the ability of knowledge flow had attracted the researchers' attention in the empirical regression analysis and dynamic investigation of knowledge network. It was found that the application effect of artificial intelligence technology on scientific and technological innovation increment and knowledge transformation was significant [2]. Maity [3] found that artificial intelligence 
technology enabled knowledge systems to effectively identify innovation coupling factors, and the ability of technology release would gradually increase after innovation alliance, so as to realize the progressive, updating, and dynamic communication of innovation system [3]. Colombelli, Quatraro [4] believed that intelligent technology promoted the coupling effects of innovation factors in the process of knowledge flow. Knowledge innovation management could be built on the premise of mutual trust, promote knowledge network diffusion, realize knowledge interaction, and drive scientific and technological innovation through knowledge platform sharing [4]. Assunta et al. [5] and Vajpayee and Ramachandran [6] believed that intelligent identification of resources and decentralized acquisition of innovation factors would ultimately increase the efficiency of knowledge innovation management $[5,6]$. In the process of promoting the release of knowledge, the higher the degree of intelligence, the faster the speed of knowledge innovation. In the mode of industry university complementary, the complementary of industry university resources promoted knowledge storage and sharing, and the innovation risk of innovation organization would be relatively reduced. Zerfass et al. [7] found that artificial intelligence had constructed a new mode of knowledge demand and knowledge supply and systematically optimized the knowledge network, knowledge gradient, and risk mechanism of knowledge innovation management through intelligent virtual technology organization. They believed that AI would orientate the knowledge flow, knowledge absorption, knowledge spillover, knowledge diffusion, knowledge transmission, and knowledge application [7]. The innovation, integration, application, and recreation of knowledge could be multiply linked with intelligent methods. George et al. [8] analyzed the aggregation of knowledge resources and the improvement of the efficiency of innovation policy sources. The application policy sources were the high-level goals of knowledge flow, and artificial intelligence technology played a facilitating role in the process of knowledge diffusion and release [8]. AI application have be further subdivided into the bottom general algorithm and the upper application from the technical point of view. For instance, the intellectual property protection methods could be designed, respectively, according to their various technical attributes. For the general application focusing on solving the technical problem of "how to simulate innovation," the patent management system should be used to promote relevant technology research and information exchange. AI application has strong innovation leading way; it can be applied by comprehensive use of diversified institutional tools including patents, copyrights, and IP trades. The knowledge network played a foundation role in new scientific discoveries and new industrial directions. With the deepening adjustment of knowledge application network, its uncertainty and risk would be gradually increasing. Therefore, the intelligent dynamic knowledge network played a key role.

This research applied the synergy model to explore the key effects and essential factors of AI on knowledge innovation management. From the preliminary studies, the virtual organization had further extended in the knowledge network through the evolution of the operation mode of intelligent innovation subject. By building an intelligent knowledge sharing team or cross-region organizations, it was conducive to knowledge transmission, application, and stimulation of R\&D potential. Litau [9] proposed that innovation management should be based on the knowledge flow efficiency of the innovation subsystem. They realized the high-quality knowledge innovation achievements needed sufficient knowledge aggregation network, and the independent innovation subjects were very limited [9]. The interaction and cooperation among innovation subjects were completed through intelligent technology, so as to better release knowledge resources and complementary role. Knowledge innovation management should carry out the construction of cross regional or cross organizational innovation network. By intelligent identification, progression, sharing and diffusion, knowledge network could be continuously updating and adjusted in the dynamic integration of collaborative innovation. Fotis and Maria [10] reorganized the characteristics of knowledge flow and believed that the higher the knowledge intensity of collaborative innovation, the higher the level of innovation and the stronger the relevance of the subsystem in the knowledge value chain [10]. The collaborative innovation models and intelligent development model had obvious advantages in knowledge flow and stock, which was one of the important models of knowledge absorption and release. Intelligent cooperation and joint project research could strengthen the construction of knowledge network of innovation subsystem and improve the effectiveness of knowledge innovation.

To sum up, the existing researches mainly analyzed the macroinfluencing effects of artificial intelligence for knowledge innovation management. Knowledge innovation management system can be divided into foundation layer, technology layer, and application layer, and the number of users in application layer is large, while the number of users in technology layer and foundation layer is relatively small. This study focuses on the application scenarios of intelligent knowledge management according to the hierarchical distribution, formulates the map of knowledge industry applying artificial intelligence technology, and subdivides the layout of artificial intelligence in knowledge management for innovation industry in combination with the characteristics of knowledge innovation management in innovation-driven region. Therefore, there is still a lot of research space to promote the beneficial adjustment of innovation ways by combining the artificial intelligence resource with knowledge innovation system and deepening the agglomeration effect of artificial intelligence for knowledge innovation management. As a result, this paper intends to break through the previous empirical ideas, start from the capability elements, environmental elements, and dynamic elements of knowledge innovation management, introduce various dimensional variables, including knowledge flow, knowledge stock, knowledge network, and knowledge mobility, accurately identify the endogenous regulatory effects of artificial intelligence on knowledge innovation management, analyse the knowledge elements and network construction by AI, and explore collaborative driving effects 
and factors of artificial intelligence. This is an important empirical study for deepening the agglomeration impact of knowledge element resources and the development of intelligent industry.

\section{Methodology}

Knowledge innovation management can be characterized in terms of knowledge flow management, knowledge stock management, knowledge mobility management, and knowledge network management. The study has considered the dissipation characteristics of knowledge innovation management, and the applicability of synergy model and adiabatic elimination method is to mainly reflect the differentiation of the subsystems within the knowledge management system. When the index coefficient is close to the linear state value, it will highlight the leading factors in the measurement process. It is conducive to strict measurement and test analysis. In addition, the recognition function is obtained by adiabatic elimination method, and then the evolution equations of knowledge innovation management are deduced. According to the distribution characteristics of artificial intelligence application, the core area of Guangdong, Hong Kong, and Macao Great Bay area is selected as the main source of the sample; the influence of extreme values and abnormal intervention values is removed. Therefore, 3765 samples are confirmed, which are development institutions, colleges, universities and high-tech enterprises. The data are from China Science and Technology Statistics Database from 2011 to 2020.

2.1. Assumptions. Knowledge management system consists of knowledge flow elements, stock elements, environmental elements, and network related elements. These innovative elements are efficiently integrated and applied by artificial intelligence to make a series of intelligent decisions, which has become the core context of knowledge intelligence management. At present, many intelligent decision-making systems, including supply chain management system (SCM), customer relationship management system (CRM), enterprise resource planning system (ERP), these systems are based on flow factors, stock factors, and environmental factors to make intelligent decisions. In addition, the important pillar of knowledge innovation management is the practical utility of knowledge dissemination, adoption, and application. Therefore, knowledge innovation management can be refined into knowledge flow management, knowledge stock management, knowledge mobility management, and knowledge network management. The collaborative effect of AI on knowledge stock management is represented as value segmentation of intelligent application. Innovation contribution of intelligent application is for the impact of AI on knowledge mobility management. The reaction of AI on knowledge network management is represented as intelligent application interaction strength [11]. Thus, the main control variables for judging the effects of AI on knowledge innovation management include intellectual property transaction rate (IPTR), intelligent output rate (IOVR), original innovation productivity rate (OIPR), and intelligent cooperative output rate (ICOR), as shown in Figure 1.

In Figure 1, knowledge flow is an important factor in the formation of comparative advantage of knowledge innovation management subjects. The absorption and diffusion of knowledge by innovation subject are closely related to knowledge flow. The study assumes that technological innovation process needs to dynamically identify the innovation capability elements, power elements, and environmental elements of the knowledge innovation management subject. Artificial intelligence technology can improve the progressive effect of the above factors to a greater extent, affect the benchmark variables of knowledge policy sources, and have a penetrating impact on the surface runoff and deep runoff of the knowledge system. Knowledge flow has high abstract characteristics in the flowing environment. In the process of knowledge absorption and diffusion, artificial intelligence technology dynamically identifies and automatically absorbs innovative elements with different characteristics and gradually forms knowledge gradient advantages in the process of integrating multiple knowledge elements. Finally, it is manifested in the improvement of knowledge innovation management performance through cumulative superposition path, deep runoff, and aggregation. Cross domain and cross organization produce dissipation, but mutiknowledge interaction produces aggregation [12]. The role of artificial intelligence technology is particularly prominent, which greatly improves the knowledge flow, promotes the enhancement of knowledge adhesion, and finally forms the value segmentation layer of knowledge innovation management. In addition, artificial intelligence technology can induce knowledge flow to reach subsisting value in the automatic recognition system. At this time, the innovation value of knowledge will grow rapidly in a certain period of time and promote the efficiency of knowledge innovation management. Therefore, it is important to evaluate the multidimensional variables of knowledge innovation management and monitor the influence of artificial intelligence on knowledge flow. Therefore, the study assumes that artificial intelligence has a positive correlation with knowledge flow. That means the more comparative the advantages of intelligent applications, the higher the intellectual property transaction rate.

2.2. Synergy Model. Knowledge management is mainly measured by the total number and degree of network nodes. The more network nodes and higher degree, the more complex the context of knowledge transfer, the larger the scale of knowledge stock, and the richer the innovation resources driven by knowledge transfer. The study is conducive to accurately identify the fast and slow relaxation variables of $\mathrm{AI}$ in the process of knowledge innovation management [13]. The strength of collaborative interaction of knowledge innovation management system is measured by $S_{1}$ and $S_{2}$, the parameters are represented by $r$ and $\rho$, and $\sigma_{1}$ and $\sigma_{2}$ represent the damping coefficient, so the synergy model is expressed as follows: 


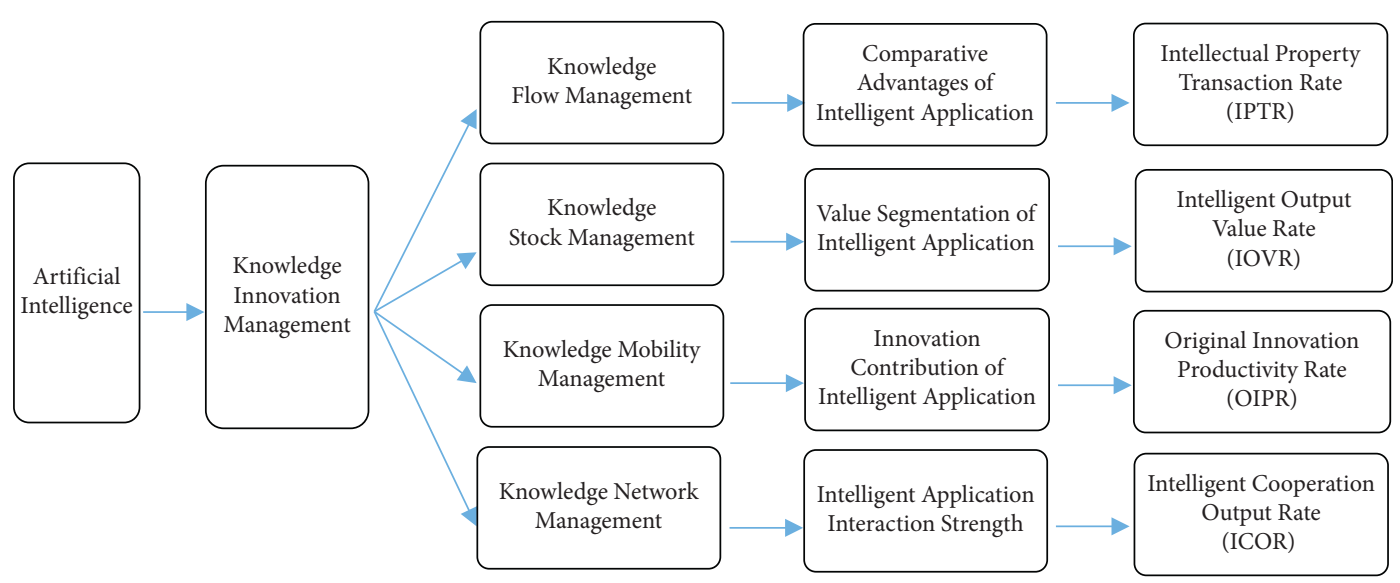

FIGURE 1: Effect diagram of AI on knowledge innovation management.

$$
\begin{aligned}
& \dot{S}_{1}=-\sigma_{1} S_{1}-r S_{1}^{2}+m, \\
& \dot{S}_{2}=-\sigma_{2} S_{2}-\rho S_{1} S_{2}+n .
\end{aligned}
$$

Obtaining identification function by adiabatic elimination method, $S_{1} \approx 0, S_{2} \approx 0$; then

$$
\begin{aligned}
& S_{1}(t) \approx \frac{r}{S_{2}} S_{1}^{2}(q), \\
& S_{2}(t) \approx \frac{\rho}{S_{1}} S_{2}^{2}(q) .
\end{aligned}
$$

The regression model is expressed as follows:

$$
M=\frac{1}{2 \sigma_{1} \sigma_{2} S_{1}^{2}}+\frac{r \rho}{4 \sigma} S_{2}^{4} .
$$

It is worth noting that artificial intelligence technology can cooperate with progressive knowledge system when the number of knowledge nodes and degrees increase. When the knowledge stock reaches a certain state value, the knowledge system will be more intensive and responsive with the continuous increase of nodes, that is, the more nodes, the more complex the network structure, the more obvious the scale benefit of the stock, and the more space to promote the efficiency of knowledge transmission. [14]. Therefore, it is very important to measure and analyse the equilibrium value of knowledge stock for the improvement of transmission efficiency. From (1) and (2), the absorption and diffusion mode of knowledge innovation management is the main subject. $-\sigma_{1} S_{1}-r S_{1}^{2}+m$ is necessary to distinguish the knowledge network structure and knowledge circulation environment and examine the storage capacity and knowledge network architecture of knowledge progressive system. $-\sigma_{2} S_{2}-\rho S_{1} S_{2}+n$ indicates the network node potential difference and knowledge flow inertia, which are comprehensively identified. Artificial intelligence plays a role in improving the interaction strength in the process of knowledge flow, which is usually reflected in the feedback of joint technological innovation projects or in the construction of cooperative engineering tasks. Therefore, this study obtains identification function by adiabatic elimination method. To make $S_{1} \approx 0, S_{2} \approx 0$, the study denotes (3) and (4), and the embedding degree of artificial intelligence determines the sorting effect of knowledge innovation interaction, especially in the efficient absorption of new knowledge and new skills, which can further enhance the interaction strength of knowledge system (3) and (4) applied in the cooperative contribution of knowledge subject. For knowledge stock management, artificial intelligence promotes the formation of obvious knowledge value segmentation and comparative advantage and provides continuous improvement for knowledge innovation management system. Therefore, the study assumes that artificial intelligence has a significant positive impact on knowledge stock. That means the more comparative the advantages of intelligent applications, the higher the intellectual property transaction rate.

As for the synergy model of AI on knowledge mobility management, the study assumes that knowledge mobility can reflect the basic conditions of the core value of knowledge innovation management system. It plays an important role in the innovation spillover of knowledge subject, closely related to the knowledge absorption layer, diffusion layer, and application layer, and finally realizes the acquisition of new knowledge and new skills [15]. According to Keng Siau and Weiyu Wang, the value segmentation of knowledge innovation management focuses on knowledge flow ability, because the feedback effect of knowledge flow ability on innovation performance is obvious [16]. In the knowledge network structure, $\dot{S}_{1}=-\sigma_{1} S_{1}-r S_{1}^{2}+m$ and $\dot{S}_{2}=-\sigma_{2} S_{2}-\rho S_{1} S_{2}+n$ are the explicit knowledge with low penetration cost, which is usually difficult to form a higher value segmentation, and the second one is the tacit knowledge with high penetration cost. Knowledge has different transmission channels and flow capacity. It is necessary to systematically integrate and embed (3) and (4) according to the characteristics of knowledge subject. Artificial intelligence plays an automatic recognition role in the progressive process of two kinds of knowledge, which makes the knowledge flow not based on regional or organizational boundary barriers but enhances the progressive space of knowledge as the goal, highlights the performance orientation of knowledge application, dynamically adjusts to the integration mode, and produces strong knowledge flow 
ability, from knowledge recognition to absorption and then to knowledge application. The innovation value of knowledge innovation management is generated efficiently; that is, artificial AI has a positive effect on the optimization and systematic deployment of knowledge mobility management. Knowledge network is not only related to the operation of innovation mechanism and the implementation of conventions but also to the hub of innovation diffusion and aggregation. Artificial intelligence optimizes intelligence resources continuously in the knowledge network environment and lays the foundation for information absorption and transmission. The knowledge network management is the comprehensive system which is carried out by improving the way of knowledge flow and finally improves the effectiveness of knowledge policy sources. Therefore, the positive correlation between AI and knowledge network management is high.

\section{Numerical Analysis}

By using the proposed model, the research focuses on the knowledge innovation management data of science and technology innovation cluster in Guangdong, Hong Kong, and Macao. There are 3765 valid samples from research and development institutions, colleges, universities, high-tech enterprises, and science and technology leading enterprises and the data come from the upfront surveys and the database of China Science and Technology Statistics from 2011 to 2020. After removing the influence of extreme value and abnormal intervention value.

3.1. Descriptive Statistics. The variables are processed by normal distribution to determine the descriptive statistical value of the impact of artificial intelligence on knowledge innovation management, and then the correlation test and identification statistical analysis are carried out. In Table 1, the statistical analysis values of variables mainly include intellectual property transaction rate (IPTR), proportion of intelligent output value (IOVR), original innovation productivity (OIPR), and proportion of intelligent cooperation output value (ICOR).

It can be seen from Table 1 that the average value of intellectual property transaction rate (IPTR) is $19.6 \%$, the standard deviation is $17.3 \%$. The results show that the impact of AI on knowledge flow management is $19.6 \%$ on average, and the penetration of AI into knowledge flow management is up to $52.3 \%$. The mean value of OIPR is $10.2 \%$, the standard deviation is $9.7 \%$, and the maximum value is $28.7 \%$. The average value of ICOR is $34.1 \%$, the standard deviation is $28.6 \%$, and the maximum value is $73.4 \%$. It shows that in knowledge flow management, average value of AI contribution to knowledge interaction application is $10.2 \%$, and the maximum value is $28.7 \%$. In the knowledge network management, the influence of artificial intelligence on application interaction intensity is $34.1 \%$ on average, and the maximum influence value of this variable on intelligent cooperation projects is $73.4 \%$.
3.2. Pearson Correlation Test. The main control variables are further tested by person correlation to determine the correlation of $\mathrm{AI}$ to knowledge innovation management variables.

As shown in Table 2, ICOR has a significant positive correlation with the transaction rate of intellectual property at the level of $1 \%$, which indicates that knowledge network management has a positive impact on knowledge traffic management, promoting the interaction strength of intelligent applications and forming the comparative advantage of innovation. The positive correlation between OIPR and the proportion of intelligent output value is $1 \%$, which indicates that there is a significant correlation between knowledge flow management and knowledge stock management.

3.3. Identification Analysis. When the model is discretely processed, the main control variables IPTR, IOVR, OIPR, and ICOR of knowledge innovation management are used to identify the described variables, as shown in Table 3. The statistical results of IPTR identification are more significant than the test value $S(0.435)$ and $S(0.512)$, indicating that AI has a strong positive correlation with knowledge flow management and has a great impact on knowledge innovation management. The results of OIPR are significant, which are $S(0.643)$ and $S(0.356)$, indicating that AI has a strong influence on knowledge flow management. According to models (3) and (4), ICOR recognition results are significantly higher than the test value $S(0.532)$ and $S$ (0.324), indicating that the positive correlation between AI and knowledge network management is high, and it has a great impact on the output value of intelligent cooperation. From the statistical results of IOVR recognition, it is consistent with the test threshold.

According to model (5), when the penetration degree of artificial intelligence reaches $M(0.643)$, the knowledge innovation management system will be intensive with the continuous increase of knowledge flow nodes and enhance the degree of collaborative innovation, which shows that when the knowledge stock reaches the state value, the complexity of knowledge innovation management network structure will be improved, and the scale efficiency of artificial intelligence application will be more obvious, which is closely related to knowledge stock management and knowledge network management. Thus, the hypothesis is further identified.

3.4. Regression Results. By multiple linear regression, the research focuses on testing the influencing factors of knowledge flow management, knowledge stock management, knowledge flow management, and knowledge network management and empirically analyzing the mechanism of artificial intelligence on knowledge innovation management. As shown in Table 4, in the regression results of model (1), IPTR is 0.726 , which is significant at the $5 \%$ level. The results show that the application of artificial intelligence has a substantial impact on the surface runoff and deep runoff of knowledge flow management, which is conducive to enhancing the internal driving force of intellectual property application. From the regression results of model model (5), 
TABLE 1: Statistical analysis value.

\begin{tabular}{lccccccrc}
\hline Variable & $\mathrm{N}$ & Min & P25 & P50 & P75 & Max & Mean & SD \\
\hline IPTR & 3765 & 0.002 & 0.021 & 0.023 & 0.412 & 0.523 & 0.196 & 0.173 \\
IOVR & 3765 & 0.004 & 0.087 & 0.133 & 0.187 & 0.257 & 0.134 \\
OIPR & 3765 & 0.010 & 0.010 & 0.021 & 0.183 & 0.287 & 0.102 \\
ICOR & 3765 & 0.128 & 0.143 & 0.217 & 0.486 & 0.734 & 0.341 & 0.097 \\
\hline
\end{tabular}

Note. The figures are descriptive statistical values of variables. In order to overcome the influence of extreme values and outlines, the data in the table are processed with $1 \%$ Stata tail reduction.

TABLE 2: Pearson correlation coefficient.

\begin{tabular}{lccc}
\hline & IPTR & IOVR & OIPR \\
\hline IPTR & 1.000 & & \\
IOVR & $0.731^{*}$ & 1.000 & 1.000 \\
OIPR & $0.612^{*}$ & $0.552^{*}$ & $0.093^{* *}$ \\
ICOR & $0.076^{* *}$ & $0.027^{* * *}$ & 1.000 \\
\hline
\end{tabular}

Note. ${ }^{* * *},{ }^{* *}$, and ${ }^{*}$ represent significance at $1 \%, 5 \%$ and $10 \%$ levels.

TABLE 3: Identification statistics.

\begin{tabular}{llcr}
\hline Variable & Models $(1)$ and $(2)$ & Models $(3)$ and $(4)$ & Model $(5)$ \\
\hline IPTR & $S_{1}(0.437) S_{2}(0.536)$ & $S_{1}(0.347) S_{2}(0.353)$ & $(0.537)$ \\
IOVR & $S_{1}(0.422) S_{2}(0.413)$ & $S_{1}(0.439) S_{2}(0.458)$ & $M(0.643)$ \\
OIPR & $S_{1}(0.643) S_{2}(0.356)$ & $S_{1}(0.543) S_{2}(0.548)$ & $M(0.622)$ \\
ICOR & $S_{1}(0.643) S_{2}(0.564)$ & $S_{1}(0.536) S_{2}(0.564)$ & $M$ \\
Constant & $S_{1}(0.427) S_{2}(0.243)$ & $S_{1}(0.232) S_{2}(0.325)$ & $M(0.654)$ \\
Inspection value & $S_{1}(0.435) S_{2}(0.512)$ & $S_{1}(0.532) S_{2}(0.324)$ & $M$ \\
Critical value & $S_{1}(0.513) S_{2}(0.414)$ & $S_{1}(0.427) S_{2}(0.534)$ & $(0.432)$ \\
$\mathrm{N}$ & 3765 & 3765 & $(0.374)$ \\
\hline
\end{tabular}

Note. The figures are the identifiable statistical values of the main control variables.

TABLE 4: Multiple linear regression value.

\begin{tabular}{lccccc}
\hline Variable & Model $(1)$ & Model $(2)$ & Model $(3)$ & Model $(4)$ & Model $(5)$ \\
\hline IPTR & $0.726^{* *}(1.36)$ & $0.827(2.25)$ & $0.673(2.40)$ & $0.543(1.57)$ & $0.652(1.72)$ \\
IOVR & $-0.392(0.34)$ & $0.472^{* *}(0.41)$ & $-0.037^{* *}(0.26)$ & $0.325^{* *}(-0.29)$ & $0.414^{* * *}(0.31)$ \\
OIPR & $0.416(0.27)$ & $0.328(0.37)$ & $0.542^{* * *}(0.21)$ & $0.612^{* *}(0.42)$ & $0.546(0.52)$ \\
ICOR & $0.206(0.83)$ & $0.319^{* *}(0.43)$ & $0.397^{* *}(-0.06)$ & $0.339(0.72)$ & $0.643^{* *}(0.51)$ \\
\hline
\end{tabular}

Note. The data in brackets are $T$ values; ${ }^{* *},{ }^{*}$, and ${ }^{*}$ represent significant differences at $1 \%, 5 \%$, and $10 \%$ levels.

OIPR is 0.546 and IOVR is 0.414 , and the regression values are significant at $1 \%$ level. The results show that artificial intelligence plays a closely related role in technology spillover of knowledge industry and plays a leading role in acquiring new skills and knowledge, which is mainly reflected in the dynamic improvement of intelligent output value, while continuously strengthening the ability of knowledge absorption and diffusion, which plays a key role in guiding the innovation strategy of intelligent industry. In addition, the regression results of model (4) showed that IOVR is 0.325 and ICOR is 0.339 , which are significant at the $5 \%$ level, indicating that AI can stimulate the increase of knowledge stock and knowledge network diffusion and promote the performance of intelligent cooperative innovation. According to the regression results of model (2), IOVR is 0.472 and ICOR is 0.319 , and the regression values are significant at $1 \%$ level. Meanwhile, the regression results of model (3) are ICOR $(0.397)$ and IOVR $(-0.037)$. The data illustrate that artificial intelligence has obvious effects on intelligence output and intelligent cooperation. With the continuous improvement of auxiliary and complementary technologies for the application of artificial intelligence technology, the level of regional intelligent manufacturing can be essentially improved. Intelligent cooperation has promoted and improved the scientific and technological innovation resources, industrial scale, and industrial base. Artificial intelligence improves the valueadded space of knowledge diffusion and knowledge application, strengthens the deep runoff knowledge transmission, stimulates the ability elements of intelligent innovation policy source, and forms obvious innovation advantages of intelligent management.

3.5. Robust Test. In order to verify the reliability of the conclusion, multiple sample comparison is used for robustness test. The validation results of the three sample sets are basically consistent with the regression results, so the research conclusions are robust and reliable, as shown in Table 5. 
TABLE 5: Robustness value.

\begin{tabular}{lccccc}
\hline Variable & Sample I & Sample II & Sample III & Variance & SE \\
\hline IPTR & 6.327 & $6.431^{* *}$ & 5.327 & -0.017 & 0.138 \\
IOVR & $2.426^{* *}$ & $3.420^{* *}$ & $2.352^{* *}$ & 0.341 & 0.371 \\
OIPR & $4.972^{* *}$ & 3.328 & $4.523^{* * *}$ & $0.214^{* *}$ & 0.043 \\
ICOR & $3.432^{* * *}$ & $2.731^{* * *}$ & 5.741 & $0.064^{* *}$ & 0.273 \\
\hline
\end{tabular}

Note. Three samples are randomly selected from CSTD; ${ }^{* *},{ }^{*}$, and ${ }^{*}$ represent significant differences at $1 \%, 5 \%$, and $10 \%$ levels.

\section{Conclusion}

Based on the statistical data of knowledge innovation management in Guangdong, Hong Kong, and Macao Bay Area from 2011 to 2020, this paper empirically tests the collaborative driving effects and key factors of artificial intelligence on knowledge innovation management by using synergy model and adiabatic elimination method. The following conclusions can be drawn from this study.

Artificial intelligence promotes the formation of the original technological advantages of knowledge innovation subjects and has obvious automatic recognition function for new knowledge and new skills, which stimulates the main internal transmission power of knowledge innovation management. The application of artificial intelligence is closely related to knowledge network management, especially in the stage of innovation performance output of intelligent industry. There is a high correlation between AI and knowledge flow management. Intelligent technology determines the energy level of original innovation and the progressive achievements of advanced technology, which mainly comes from the deep knowledge of innovation system. Knowledge network affects the transmission efficiency and retention ratio of deep runoff knowledge. The penetration ratio of artificial intelligence determines the cooperation space of intelligent industry and the direction of integrating knowledge application, which plays an important role in guiding the industrial innovation system. The construction of knowledge flow environment and flow network is conducive to the formation of higher primary innovation comparative advantage and value segmentation layer and promotes the overall optimization of knowledge innovation management.

\section{Data Availability}

Data sharing not applicable to this article as no datasets were generated or analyzed during the current study.

\section{Conflicts of Interest}

The authors declare no conflicts of interest.

\section{Acknowledgments}

This research was supported by the Guangdong Basic and Applied Basic Research Foundation (Grant: 2019A1515110695), Guangdong Education and Teaching Reform Project (Grant: 2018yjjg002), and Key Discipline Group of Business Administration in City College of Dongguan University of Technology.

\section{References}

[1] L. F. D. C. Botega and J. C. Da Silva, "An artificial intelligence approach to support knowledge management on the selection of creativity and innovation techniques," Journal of Knowledge Management, vol. 24, no. 5, pp. 1107-1130, 2020.

[2] F. T. Vikingur, I. Helgi Thor, J. H. Ingi, and J. Hildur, "An authoritative study on the near future effect of artificial intelligence on project management knowledge areas," Sustainability, vol. 13, no. 4, pp. 2345-2354, 2021.

[3] S. Maity, "Identifying opportunities for artificial intelligence in the evolution of training and development practices," The Journal of Management Development, vol. 38, no. 8, pp. 651663, 2019.

[4] A. Colombelli and F. Quatraro, "New firm formation and regional knowledge production modes: Italian evidence," Research Policy, vol. 47, no. 1, pp. 139-157, 2018.

[5] D. V. Assunta, P. Rosa, R. Hassan, and E. Octavio, "Artificial intelligence and business models in the sustainable development goals perspective: a systematic literature review," Journal of Business Research, vol. 121, pp. 283-314, 2020.

[6] A. Vajpayee and K. K. Ramachandran, "Reconnoitring artificial intelligence in knowledge innovation management," International Journal of Innovative Technology and Exploring Engineering, vol. 8, no. 7c, pp. 114-117, 2019.

[7] A. Zerfass, J. Hagelstein, and R. Tench, "Artificial intelligence in communication management: a cross-national study on adoption and knowledge, impact, challenges and risks," Journal of Communication Management, vol. 24, no. 4, pp. 377-389, 2020.

[8] S. George, D. Karapistolis, and A. Vafeiadis, "Marketing decision support using artificial intelligence and knowledge modeling: application to tourist destination management," Procedia Social and Behavioral Sciences, vol. 175, pp. 106-113, 2015.

[9] E. Y. Litau, "Scoring method as applied to innovation project evaluation for startup support," Entrepreneurship and Sustainability Issues, vol. 7, no. 4, pp. 2978-2990, 2020.

[10] K. Fotis and K. Maria, "Artificial intelligence and business strategy towards digital transformation: a research agenda," Sustainability, vol. 13, no. 4, pp. 2025-2036, 2021.

[11] A. Di Vaio, R. Palladino, R. Hassan, and O. Escobar, "Artificial intelligence and business models in the sustainable development goals perspective: a systematic literature review," Journal of Business Research, vol. 121, no. 121, pp. 283-314, 2020.

[12] H. F. L. Chung, D. A. Yen, and C. L. Wang, “The contingent effect of social networking ties on Asian immigrant enterprises' innovation," Industrial Marketing Management, vol. 88, no. C, pp. 414-425, 2020.

[13] E. R. De Aro and G. Perez, "Identification of dynamic capabilities in open innovation," Innovation \& Management Review, vol. 18, no. 2, pp. 118-128, 2021.

[14] A. Fieras and A. Tarik, "The effect of strategic human resource and knowledge management on sustainable competitive 
advantages at Jordanian Universities: the mediating role of organizational innovation," Sustainability, vol. 13, no. 15, pp. 8445-4448, 2021.

[15] J. Aarstad, O. A. Kvitastein, and S.-E. Jakobsen, "Related and unrelated variety as regional drivers of enterprise productivity and innovation: a multilevel study," Research Policy, vol. 45, no. 4, pp. 844-856, 2016.

[16] K. Siau and W. Wang, "Artificial intelligence, machine learning, automation, robotics, future of work and future of humanity: a review and research agenda," Journal of Database Management, vol. 30, no. 1, pp. 61-79, 2019. 